\title{
Effect of dry-sausage starter culture and endogenous yeasts on Aspergillus westerdijkiae and Penicillium nordicum growth and OTA production
}

\author{
Sana Meftah a, Salwa Abid ${ }^{b}$, Teresa Dias ${ }^{a}$, Paula Rodrigues a, * \\ ${ }^{a}$ Mountain Research Centre (CIMO), ESA, Polytechnic Institute of Bragança, Campus de Santa Apolónia, 5300 -253 Bragança, Portugal \\ ${ }^{\mathrm{b}}$ Laboratory for Research on Biologically Compatible Compounds, Faculty of Dentistry, Rue Avicenne, 5019 Monastir, Tunisia
}

\section{A R T I C L E I N F O}

\section{Article history:}

Received 24 March 2017

Received in revised form

25 July 2017

Accepted 24 August 2017

Available online 31 August 2017

\section{Keywords:}

Ochratoxigenic fungi

Ecophysiology

Food safety

Fermentation

Meat products

\begin{abstract}
A B S T R A C $T$
Processed meat products frequently suffer from fungal and mycotoxin contamination, mostly ochratoxin A (OTA). Penicillium nordicum is considered responsible for this contamination, but Aspergillus westerdijkiae has recently been associated with high levels of OTA in meat products.

Several biocontrol agents have been tested against $P$. nordicum growth and OTA production in meat products, but $A$. westerdijkiae has not been considered. The aim of this work was to evaluate in vitro the effect of a commercial starter culture used in sausage fermentation and of sausage-native yeasts on OTA production by $A$. westerdijkiae, as compared with the highly studied $P$. nordicum, in meat-based culture media.

Four representative yeasts isolated from dry-cured sausage and a commercial starter culture were coinoculated with both fungi in different meat-based media, under varying conditions. Fungal growth was determined by measuring colony diameter, and OTA production was quantified by HPLC-FLD.

A. westerdijkiae was significantly stimulated to produce OTA under all tested conditions, and, in ham, OTA production by $P$. nordicum was stimulated by co-culture with the starter culture.

In conclusion, endogenous or added microorganisms enrolled in fermentation or in biocontrol in meat products seem to exert varying responses on different ochratoxigenic fungi, thus leading to unforeseen safety problems.
\end{abstract}

๑) 2017 Elsevier Ltd. All rights reserved.

\section{Introduction}

Processed meat products such as dry-cured ham, fermented sausage and others are foods of major importance in several European countries, both nutritionally and economically. Portuguese traditional fermented cured sausages (Salpicão, Linguiça, Chouriça) are prepared from raw pork meat with salt, and ingredients such as red/white wine, garlic and chili pepper. Some curing salts like nitrite and polyphosphates can also be added. The process includes a first stage of seasoning, stuffing of the mixture in natural pork casings, and a final stage of curing (with or without smoking), from one to four weeks, depending on the product and the process. (Cadavez et al., 2016). The fermentation of these traditional products occurs naturally, by the action of characteristic endogenous

\footnotetext{
* Corresponding author.

E-mail address: prodrigues@ipb.pt (P. Rodrigues).
}

microbiota comprising essentially lactic acid bacteria (LAB), coagulase-negative staphylococci (CNS) and yeasts. Yeasts show important beneficial effects throughout curing, as they contribute with particular aroma and flavour resulting from their proteolytic and lipolytic activities (reviewed by Flores, Corral, Cano-García, Salvador, \& Belloch, 2015). Although data on the yeast dynamic and biodiversity in sausages are limited, several studies revealed the presence of some genera such as Debaryomyces, Candida, Yarrowia, Rhodotorula, Pichia and Trichosporon (Andrade, Rodriguez, Casado, Bermudez, \& Cordoba, 2010; Mendonça, Gouvêa, Hungaro, Sodré, \& Querol-Simon, 2013; Nielsen, Jacobsen, Jespersen, Koch, \& Arneborg, 2008; Romano, Capece, \& Jespersen, 2006; Samelis \& Sofos, 2003). Despite the fact that yeast population in dry-fermented meat products depends on meat flora and manufacture process, Debaryomyces hansenii has been the most frequently isolated yeast species along different manufacture processes, and nowadays selected strains are available as commercial starters. 
Dry-fermented sausages are considered safe meat products due to reduced water activity and $\mathrm{pH}$ achieved during processing and storage, which together inhibit the development of pathogenic microflora. However, due to their characteristics, they are highly exposed to mycotoxin producing fungi. Ochratoxin A (OTA) is the most significant mycotoxin found in processed meat products. Penicillium nordicum is considered to be responsible for OTA contamination of these products, as it is strongly adapted to salt and protein-rich matrices and is moderately psychrotrophic. However, another OTA-producing fungus, Aspergillus westerdijkiae (formerly known as A. ochraceus), associated with carbon-rich matrices such as cereals and coffee beans, has also been associated with meat products (Escher, Koehler, \& Ayres, 1973; Iacumin, Milesi, Pirani, Comi, \& Chiesa, 2011; Scaramuzza, Diaferia, \& Berni, 2015) and its ability to produce high amounts of OTA in such products has been proved (Iacumin, Manzano, Andyanto, \& Comi, 2017, as A. ochraceus; Vipotnik, Rodríguez, \& Rodrigues, 2017).

Because of its relevance, OTA should be reduced to the lowest possible levels in foods, and for that reason it is mandatory to develop efficient strategies to avoid it from entering the food chain. Numerous chemical, physical and biological agents can be used for food detoxification purposes (EFSA, 2009). But one of the major problems with OTA, as with other mycotoxins, remains on its high stability (Boudra, Le Bars, \& Le Bars, 1995) thus making it highly challenging to eliminate it from food products. For this reason, one of the most promising strategies under study is to prevent its accumulation by creating the best conditions to inhibit fungal growth and consequent OTA production.

Many studies have been done for the screening of yeasts, bacteria and moulds with antagonistic effect on OTA accumulation in food products, either by inhibition of fungal growth or by inhibition of OTA production. Several LAB and yeasts have been tested as biocontrol agents against $P$. nordicum growth and OTA production in meat products, mostly ham and Italian-style salami, with promising results (Andrade, Thorsen, Rodríguez, Córdoba, \& Jespersen, 2014; Iacumin et al., 2017; Rodriguez et al., 2015; Simoncini, Virgili, Spadola, \& Battilani, 2014; Virgili et al., 2012), but only one has considered the effect of these microorganisms on ochratoxigenic fungi other than P. nordicum (Iacumin et al., 2017).

The intended aims of this work were to study, in vitro, the effect of a commercial starter culture composed by LAB, CNS and $D$. hansenii and of several Portuguese dry-sausage endogenous yeasts on $P$. nordicum and A. westerdijkiae growth and OTA production ability in a Portuguese-style dry-fermented sausage-based medium. Throughout the work, some unexpected results were obtained for the matrix under study, and further studies were developed to test the influence of the matrix on the effect of biocontrol agents. For this subsequent study, two types of fermented dry-cured sausages were included. While the main interest of the work was fermented dry-cured sausage, dry-cured ham was also included, as a control, since information on OTA production ability by $P$. nordicum and $A$. westerdijkiae were available (Vipotnik et al., 2017).

\section{Materials and methods}

\subsection{Selection and identification of yeasts}

A group of 100 yeasts previously isolated from two dryfermented sausage plants and preserved at $-20{ }^{\circ} \mathrm{C}$ in $30 \%$ glycerol were grown on Potato Dextrose Agar (PDA, Liofilchem-ITALY) for 3 days at $28{ }^{\circ} \mathrm{C}$ and preliminarily grouped according to their colony and cell morphology. From these, ten yeasts were selected from morphologically distinct groups and were biochemically identified by the rapid miniaturised system API 20 C AUX System (bioMérieux, France), following supplier instructions. The following yeast species were identified: Candida albicans, Candida colliculosa, Candida krusei (2 isolates), Candida zeylanoides (2 isolates), Cryptococcus laurentii, Rhodotorula glutinis, and Rhodotorula mucilaginosa (2 isolates). From these, four strains - phenotypically identified as $C$. krusei, $C$. zeylanoides, $R$. glutinis and $R$. mucilaginosa - were further selected based on relative frequency on Portuguese dry-fermented sausage and on other similar products (Andrade et al., 2010; Mendonça et al., 2013). The identification of the four selected yeasts was confirmed molecularly by amplification and sequencing of the D1/D2 region of 26S rRNA gene. Yeasts were grown on Potato Dextrose Agar (PDA, Liofilchem-ITALY) for 3 days at $28{ }^{\circ} \mathrm{C}$, and genomic DNA extraction was performed as described by Rodrigues, Venancio, Kozakiewicz, and Lima (2009). PCR amplification was achieved with the primers NL1 (5'GCATATCAATAAGCGGAGGAAAAAG3') and NL4 (5'GGTCCGTGTTTCAAGACGG3'), as described by Kurtzman and Robnett (1998). PCR reactions were carried out in a thermal cycler BioRad Mycycler, in a final volume of $50 \mu \mathrm{L}$, containing $10 \mu \mathrm{L}$ of 5x Go-Flexi Taq $\mathrm{MgCl}_{2}$-free reaction Buffer (Promega), $1.5 \mathrm{mM}$ $\mathrm{MgCl}_{2}, 1.25 \mathrm{U}$ of Go-Flexi Taq polymerase (Promega), $200 \mu \mathrm{M}$ of each dNTP (Bioron), and $2 \mu \mathrm{L}$ of genomic DNA. PCR programme was optimized from the procedure described by Kurtzman and Robnett (1998), and was carried out as follows: initial denaturation of DNA for $4 \mathrm{~min}$ at $95{ }^{\circ} \mathrm{C}$; 34 cycles of denaturation at $95{ }^{\circ} \mathrm{C}$ for $50 \mathrm{~s}$, annealing at $55^{\circ} \mathrm{C}$ for $50 \mathrm{~s}$, and extension at $72{ }^{\circ} \mathrm{C}$ for $1 \mathrm{~min}$; and a final extension cycle at $72{ }^{\circ} \mathrm{C}$ for $7 \mathrm{~min}$. PCR products were purified with the commercial kit GF-1 PCR CleanUp Kit (Vivantis), according to manufacturer's instructions. Sequence analysis was performed on an ABI 3730xl DNA Analyzer (Applied Biosystems), by outsourcing. PCR products were sequenced in both directions, and a consensus sequence was created from the assembly of the forward and backward sequences using the package Sequencher 4.9 (Gene Codes, Ann Arbor Michigan) (Rodrigues, Santos, Venancio, \& Lima, 2011). The consensus sequences were manually adjusted by chromatogram comparison and then aligned with the NCBI GenBank database (http://www.ncbi.nlm.nih.gov/) using the BLAST algorithm. Yeast phenotypic identification was confirmed as C. krusei, C. zeylanoides, $R$. glutinis and $R$. mucilaginosa.

\subsection{Preparation of inocula}

\subsubsection{Yeasts and starter culture}

The selected yeasts were sub-cultured from stock vials onto PDA and incubated at $28{ }^{\circ} \mathrm{C}$ for 3 days. For the preparation of the preinoculum, one colony from 3 day old cultures was suspended in Potato Dextrose Broth (PDB, Liofilchem-ITALY) and incubated at $28{ }^{\circ} \mathrm{C}$ for $24 \mathrm{~h}$ in a rotary shaker $(120 \mathrm{rpm})$. Optical density of the suspension was determined by spectrophotometry at $600 \mathrm{~nm}$ wavelength (OD600). Cell concentration was estimated by using the correspondence OD600 $=1.0$ equals $3 \times 10^{7}$ cells $/ \mathrm{mL}$, as described by Day, Schneider, and Schneider (2004). An inoculum of approximately $10^{5}$ cells $/ \mathrm{mL}$ was used in all assays.

The commercial starter culture Texel ${ }^{\circledR}$ ELCE $\mathrm{Br}$ (Danisco) composed of Pediococcus pentosaceus, Lactobacillus sakei, Staphylococcus carnosus, Staphylococcus xylosus and D. hansenii - was also used for biocontrol tests. The freeze-dried starter culture $(0.01 \% \mathrm{w} /$ v) was inoculated in MRS (de Man, Rogosa, Sharpe) broth (Liofilchem-ITALY) and incubated at $37^{\circ} \mathrm{C}$ for $24 \mathrm{~h}$. An inoculum of $2 \mu \mathrm{L}$ of cell suspension per $\mathrm{mL}$ of meat extract media was used in all assays.

\subsubsection{Ochratoxigenic fungi}

Two species of OTA-producing fungi previously isolated from 
cured pork meat were used: $P$. nordicum strain PN 44 (provided by Dr. Alicia Rodríguez, University of Extremadura, Spain) and A. westerdijkiae strain 6B/131. Fungi were inoculated in Malt Extract Agar (MEA, Liofilchem-ITALY) and incubated for 7 days at $25^{\circ} \mathrm{C}$ in the dark. After incubation, $2 \mathrm{~mL}$ of $0.05 \%$ Tween 80 solution were added to the culture and spores were scrubbed to obtain a suspension. Spore suspension was adjusted to $10^{7}$ spores $/ \mathrm{mL}$ with the aid of a Neubauer counting chamber.

\subsection{Evaluation of biocontrol effect in dry-fermented sausage}

Experiments were performed in industrial dry-fermented sausage-based medium (further designated as Ind) with two different levels of $\mathrm{a}_{\mathrm{w}}$ and $\mathrm{NaCl}$. These were selected as representative of Portuguese dry-fermented sausage in the two most significant steps of production: the beginning of ripening stage (1\% $\mathrm{NaCl} ; \mathrm{a}_{\mathrm{w}} 0.98$ ) and in the final product ( $3 \% \mathrm{NaCl} ; \mathrm{a}_{\mathrm{w}} 0.95$ adjusted with 10\% glycerol) (Cadavez et al., 2016; Gonzales-Barron et al., 2015). Sausage was finely minced and lyophilized. Thirty grams of lyophilized meat product were boiled in $1000 \mathrm{~mL}$ of distilled water (3\% meat) during $30 \mathrm{~min}$ and filtered through cheese cloth. Meat extract was supplemented with $\mathrm{NaCl}$ at $1 \%\left(\mathrm{a}_{\mathrm{w}} 0.98\right)$ or with $3 \%$ and $10 \%$ glycerol ( $a_{w}$ 0.95). Two per cent of bacteriological agar (HiMedia) were added, and media were autoclaved at $121{ }^{\circ} \mathrm{C}$ for 15 min. Water activity was measured in triplicate using a water activity meter (Aqualab $4 \mathrm{TE}$ ). $\mathrm{pH}$ was measured in triplicate with a pH-meter (METTLER TOLEDO), and showed a pH ranging from 5.3 to 5.5 , which is representative of Portuguese sausages (Cadavez et al., 2016; Gonzales-Barron et al., 2015).

Media were inoculated by incorporation of $10^{5}$ cells $/ \mathrm{mL}$ of the commercial starter culture, each yeast ( $R$. glutinis, $C$. krusei, $C$. zeylanoides, $R$. mucilaginosa) and a mix of the 4 yeasts, and approximately $20 \mathrm{~mL}$ of the inoculated medium was poured into each petri dish. The mix of yeasts was used to simulate the effect of yeast community usually present in the matrix. Two $\mu \mathrm{L}$ of fungal spore suspension (equivalent to $2 \times 10^{4}$ spores/spot) were subsequently spotted on the top of the inoculated petri dishes by equidistant three-point inoculation, and were incubated at 15 and $20^{\circ} \mathrm{C}$ for 14 days. These temperatures replicate the ones existing during the ripening stage.

Fungal growth was determined throughout the incubation period every two days until coalescence, by orthogonal measurements of fungal colonies' diameter. At the end of incubation period, all Petri dishes were submitted to OTA analysis as described below. Petri dishes without test microorganisms (fungi only) were used as control. All tests were run in duplicate.

\subsection{Evaluation of the matrix influence on biocontrol effect}

After analysing the results obtained in the previous assay, the commercial starter, $R$. mucilaginosa, $C$. zeylanoides and a mix of both yeasts were selected to test the matrix influence on the biocontrol effect. For this purpose, three different matrices were used: industrial sausage (Ind) and traditional sausage (Trad) with 3\% salt and $10 \%$ glycerol added, to reduce $\mathrm{a}_{\mathrm{w}}$ to 0.95 , and dry-cured ham (Ham) with $3 \%$ salt and $2 \%$ glycerol added, to reduce $\mathrm{a}_{\mathrm{w}}$ to 0.97 (to reproduce the conditions reported by Vipotnik et al., 2017). Even though this study is based on sausage matrices, ham was introduced in this test as a positive control, since $P$. nordicum and A. westerdijkiae had been previously tested in this matrix in terms of growth and OTA production (Vipotnik et al., 2017). Coinoculations were prepared as previously described, and incubated at $20^{\circ} \mathrm{C}$ for 14 days.

\subsection{OTA analysis}

OTA was extracted from plates after 14 days of incubation. Three agar plugs were removed from the central, middle and peripheral areas of the colony, to be representative of OTA's distribution in the plate, weighted and extracted with $1.5 \mathrm{~mL}$ of methanol as previously described (Bragulat, Abarca, \& Cabañes, 2001). The agar plugs were maintained in methanol for one hour and vortexed every $15 \mathrm{~min}$. The extracts were filtered by PTFE $0.2 \mu \mathrm{m}$ syringe filters and stored at $4{ }^{\circ} \mathrm{C}$ until further analysis.

OTA was analysed by High Performance Liquid Chromatography (HPLC) system equipped with: Smartline pump (1000, Knauer, Berlin, Germany), degasser system (Smartline manager 5000), auto-sampler (AS-2057, Jasco, Easton, MD, USA), and a fluorescence detector (FP-2020, Jasco) set to $\lambda$ ex $330 \mathrm{~nm}$ and $\lambda$ em $463 \mathrm{~nm}$. Data were analysed using Clarity 2.4 Software (DataApex, Prague, Czech Republic). The chromatographic separation was performed as described in Vipotnik et al. (2017), using an isocratic elution with a C18 reverse-phase column $(100 \times 4.6 \mathrm{~mm}, 2 \mu \mathrm{m}$ pores, Merck Chromolith Performance, Darmstadt, Germany) operating at $35{ }^{\circ} \mathrm{C}$ (7971 R Grace oven). The mobile phase consisted of an isocratic programme of water: acetonitrile: acetic acid (29.5:70:0.5, v/v/v) and was pumped at $0.8 \mathrm{~mL} / \mathrm{min}$ for a total run time of $4 \mathrm{~min}$. The injection volume was $10 \mu \mathrm{L}$. Under these conditions, retention time for OTA was $2.2 \mathrm{~min}$.

Linearity, limit of detection (LOD) and limit of quantification (LOQ) were determined following the analytical method validation guidelines described by Taverniers, De Loose, and Van Bockstaele (2004). Linearity was demonstrated by three series of analyses, using 11 standard solutions with concentrations ranging from $0.05 \mathrm{ng} / \mathrm{mL}$ to $100 \mathrm{ng} / \mathrm{mL}$. The calibration curve was $y=1 \mathrm{E}-06 \mathrm{x}-$ 0.9984 and $\mathrm{R}^{2}=0.9994$. LOD and LOQ were calculated according to the following equations: $\mathrm{LOD}=\mathrm{y}_{0}+3 \times \mathrm{S}_{\mathrm{y} / \mathrm{x}}$ and $\mathrm{LOQ}=\mathrm{y}_{0}+10 \times \mathrm{S}_{\mathrm{y} /}$ $\mathrm{x}$, where $Y_{0}$ is the intercept of the regression line obtained from the calibration curve ( $\mathrm{Y} 0=-0.9984)$, and $S_{y / x}$ is the standard error of the slope $\left(S_{y / x}=0.5374\right)$. LOD and LOQ were calculated as $0.6 \mathrm{ng} / \mathrm{mL}$ and $4.3 \mathrm{ng} / \mathrm{mL}$, respectively. OTA was quantified by gram of agar, taking into consideration the weight of the agar plugs used for extraction.

\subsection{Statistical analysis}

Statistical analysis was performed using IBM $^{\circledR}$ SPSS $^{\circledR}$ Statistics v.22.0 software (Armonk, NY: IBM Corp.). For the comparison of means, samples were first tested for normal distribution by Shapiro-Wilk test $(\mathrm{n}<30)$ and for homogeneity of variances by Levene's test. Since samples generally followed these criteria, tstudent test and One-way ANOVA were used for comparison of means for 2-level variables and for more than 2-level variables, respectively. Two-way ANOVA was used to test interaction between two factors Post-hoc analyses were performed with Dunett test (to create confidence intervals for differences between the mean of each factor level and the mean of a control group). In all cases, statistical significance was established at $p \leq 0.05$.

\section{Results and discussion}

\subsection{Evaluation of biocontrol effect in dry-fermented sausage}

This study aimed to determine the effect of a selected group of microorganisms on growth and OTA production by $P$. nordicum and A. westerdijkiae. For that purpose, the four selected sausage-native yeasts, a mix of those yeasts and a starter culture commercially used in sausage fermentation and curing process were coinoculated with each fungus under two different temperatures 
(15 and $20^{\circ} \mathrm{C}$ ) and two salt concentrations ( 1 and $3 \%$ ).

The results of fungal growth (colony diameter) in Ind media with $1 \%$ and $3 \% \mathrm{NaCl}$ incubated at 15 and $20^{\circ} \mathrm{C}$ are represented in Fig. 1.

After 14 days of incubation, a significant inhibition effect was detected by all yeasts and yeast mix on $P$. nordicum and A. westerdijkiae growth under all tested conditions. On the other hand, starter culture showed a variable effect on fungal growth, with generally no significant effect of growth inhibition. These results confirm the inhibitory effect of yeasts on $P$. nordicum growth already reported by others. In previous researches, several yeasts such as Hyphopichia burtonii, D. hansenii and C. zeylanoides were reported as strong inhibitors of $P$. nordicum growth in dry-cured ham and dry fermented sausage (Andrade et al., 2014; Iacumin et al., 2017; Nuñez et al., 2015; Virgili et al., 2012). This reduction in fungal growth could be due to competition for nutrients and space (Hernández-Montiel, Ochoa, Troyo-Diéguez, \& LarraldeCorona, 2010), production of hydrolytic enzymes, killer toxins (Masih \& Paul, 2002) or secretion of volatile compounds (Fialho, Toffano, Pedroso, Augusto, \& Pascholati, 2009; Masoud, Poll, \& Jakobsen, 2005; Taczman-Brückner, Mohácsi-Farkas, Balla, \& Kiskó, 2005).

The mechanisms of action of starter culture are not well understood. The commercial starter culture is generally used in the fermentation process of meat products, and is composed of P. pentosaceus, L. sakei, S. carnosus, S. xylosus and D. hansenii. Species of genera Pediococcus and Lactobacillus have been studied as potential biocontrol agents against fungi and mycotoxins in several food matrices, with inhibiting effects being observed (Abrunhosa et al., 2014; Ngang et al., 2015; Pereira et al., 2016). D. hansenii has also previously shown ability for inhibition of fungal growth
(Andrade et al., 2014; Iacumin et al., 2017; Simoncini et al., 2014; Virgili et al., 2012).

In terms of OTA production, no detectable OTA was produced by $P$. nordicum, under any of the test conditions. This fact was unexpected, since this strain was isolated from Spanish ham, and has been reported as OTA producer in meat-based media (Vipotnik et al., 2017). It has been demonstrated that the fungus was able to grow and produce high amounts of OTA in dry-cured ham-based medium under a wide range of conditions (Vipotnik et al., 2017). The inability of the fungus to produce OTA in sausage-based media is maybe due to the composition of this meat product, either by the effect of natural composition (lipid and protein contents) or by the effect of added compounds such as wine, garlic, red pepper and polyphosphates (Gonzales-Barron et al., 2015; Suarez, Frison, de Basilico, Rivera, \& Reinheimer, 2005; Suarez, Tremmel, Rivera, Reinheimer, \& Meinardi, 2012), which are not present in drycured ham, and might have an inhibitory effect on OTA production ability.

The results of OTA production by A. westerdijkiae after 14 days of incubation in Ind media with $1 \%$ and $3 \% \mathrm{NaCl}$ at 15 and $20{ }^{\circ} \mathrm{C}$ are represented in Fig. 2.

As with $P$. nordicum, A. westerdijkiae growing under axenic conditions was unable to produce detectable amounts of OTA in Ind media, at both 15 and $20^{\circ} \mathrm{C}$.

At $15{ }^{\circ} \mathrm{C}$, amounts of OTA at very low concentrations (max. $70 \mathrm{ng} / \mathrm{g}$ ) were produced by $A$. westerdijkiae only at $3 \% \mathrm{NaCl}$ and in co-culture with $R$. mucilaginosa, $R$. glutinis and starter culture. On the other hand, when incubated at $20^{\circ} \mathrm{C}$, OTA production by this fungus was significantly stimulated by all co-cultures in both media, with $R$. mucilaginosa being the most inducing agent, resulting in

\section{P. nordicum}
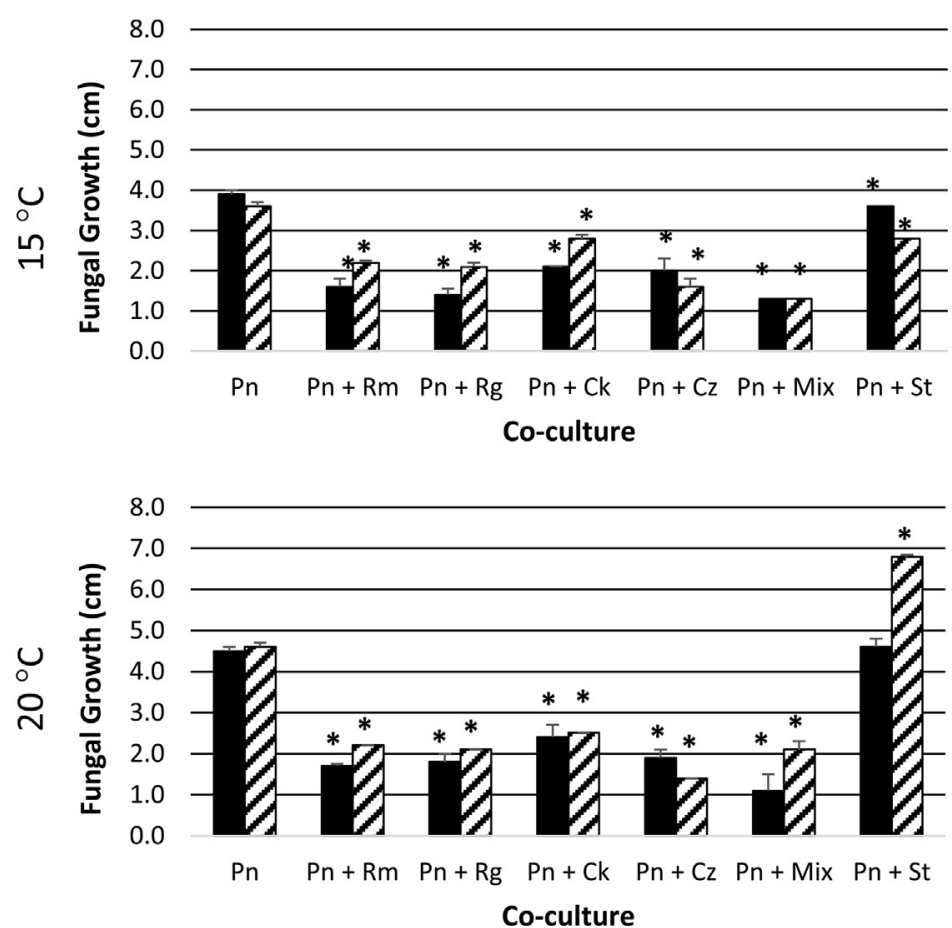

\section{A. westerdijkiae}

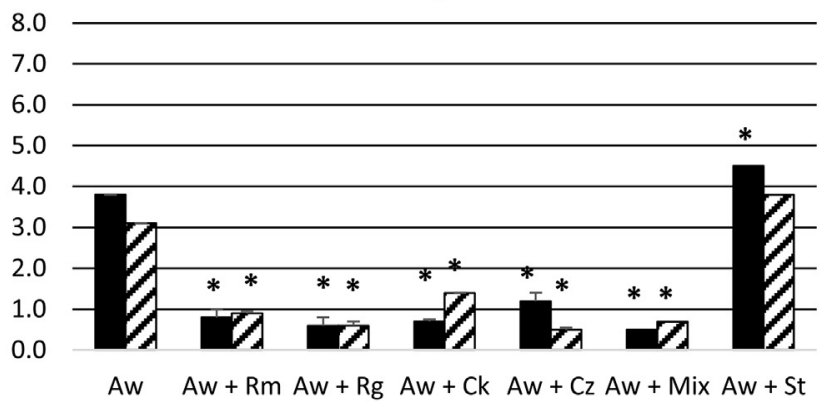

Co-culture

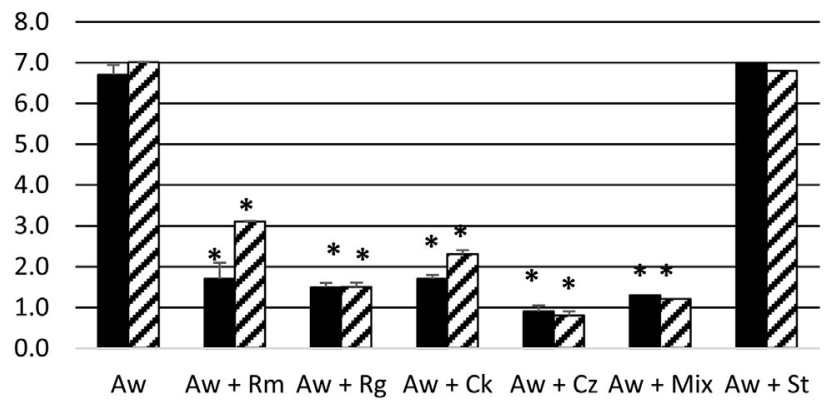

Co-culture

$1 \% \mathrm{NaCl}$ घ3\% $\mathrm{NaCl}$

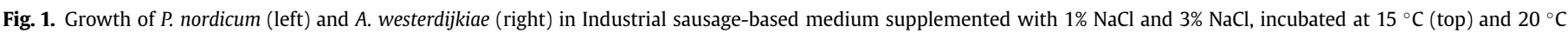
(bottom), after 14 days of incubation. In all cases, results are the average of six replicates; standard deviation is indicated as vertical thin lines.

Pn: P. nordicum; Aw: A. westerdijkiae; Rm: R. mucilaginosa; Rg: R. glutinis; Ck: C. krusei; Cz: C. zeylanoides; Mix: mix of the four yeasts; St: starter culture.

*Significantly different from control (fungus only), $p \leq 0.05$ 
$15^{\circ} \mathrm{C}$

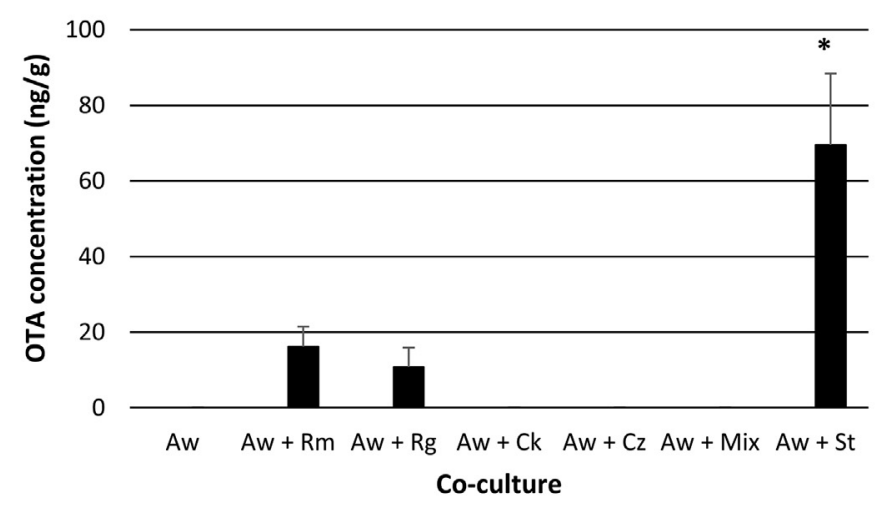

$20^{\circ} \mathrm{C}$

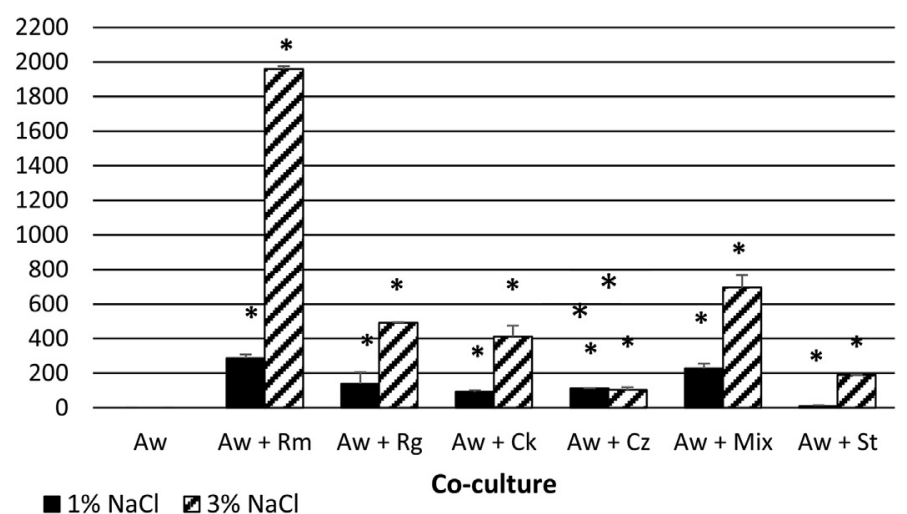

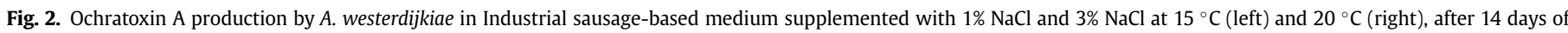

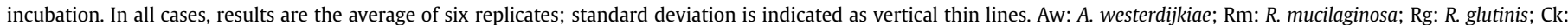
C. krusei; Cz: C. zeylanoides; Mix: mix of the four yeasts; St: starter culture.

*Significantly different from control (fungus only), $p \leq 0.05$.

extremely high levels of OTA (1959 ng/g).

Although $A$. westerdijkiae is traditionally considered a strong contaminant of carbon-rich plant-based products (Gil-Serna, Patiño, Cortés, González-Jaén, \& Vazquez, 2015), it can also contaminate dry-cured meat products. Escher et al. (1973) reported the presence of the fungus (as A. ochraceus) in cured ham samples contaminated with OTA, and Scaramuzza et al. (2015) found it in Italian meat product processing plants. Iacumin et al. (2011) found that $34 \%$ of casings of Italian sausages showing high levels of OTA were contaminated with A. ochraceus (possibly A. westerdijkiae), and is able to produce high amounts of OTA (Iacumin et al., 2017, as A. ochraceus; Vipotnik et al., 2017).

The observed OTA enhancement effect of yeasts and starter culture was also unexpected, mostly because those microorganisms have been previously tested and reported as potential biocontrol agents for other OTA-producing species on different matrices (Andrade et al., 2014; Gerez, Dallagnol, Ponsone, Chulze, \& Valdez, 2014; Gil-Serna, Patiño, Cortés, González-Jaén, \& Vazquez, 2011; Iacumin et al., 2017; Nuñez et al., 2015; Pereira et al., 2016; Simoncini et al., 2014; Virgili et al., 2012).

Even though microorganisms have inhibited $A$. westerdijkiae growth (Fig. 1), it should not be assumed that OTA would also be repressed. In fact, studies of $A$. westerdijkiae ecophysiology in plantderived products (coffee, grapes, paprika, barley, maize and anise; Gil-Serna et al., 2015) and dry-cured ham-based media (Vipotnik et al., 2017) found no correlation between fungal growth and mycotoxin production. Growth inhibition can be considered a stress factor to the fungus, which potentially results in the activation of the secondary metabolism as a sub-lethal stress response, with consequent increased OTA production. In fact, when studying fungicide effect on fungal growth and mycotoxin production, Schmidt-Heydt, Stoll, \& Geisen, (2013) detected increased production of mycotoxins under growth limiting treatments, suggesting that the fungal adaptation process to sub-lethal stress may include an upregulation of secondary metabolism as an early adaptive response.

The results of statistical analyses of the influence of two temperatures $\left(15\right.$ and $\left.20^{\circ} \mathrm{C}\right)$ and salt concentration/ $\mathrm{a}_{\mathrm{w}}\left(1 \% \mathrm{NaC} / 0.98 \mathrm{a}_{\mathrm{w}}\right.$ and $3 \% \mathrm{NaCl} / 0.95 \mathrm{a}_{\mathrm{w}}$ ) on fungal growth and OTA production are represented in Table 1 . Interactions between available water and temperature are fundamental because they represent the twodimensional niche in which fungi may be able to germinate, grow and actively compete for the allocation of the available resources
Table 1

Statistical analyses of the influence of temperature, water activity and the interaction of both on P. nordicum (Pn) growth and A. westerdijkiae (Aw) growth and OTA production $(p<0.05)$.

\begin{tabular}{lllll}
\hline Species & Test variable & Factor & Statistical set & $p$-value \\
\hline Pn & Growth & Temperature & One way ANOVA & 0.010 \\
& & $\mathrm{a}_{\mathrm{w}}$ & One way ANOVA & 0.122 \\
& & Temperature $\times \mathrm{a}_{\mathrm{w}}$ & Two way ANOVA & 0.277 \\
Aw & Growth & Temperature & One way ANOVA & 0.000 \\
& & Aw & One way ANOVA & 0.923 \\
& \multirow{3}{*}{ OTA } & Temperature $\times \mathrm{a}_{\mathrm{w}}$ & Two way ANOVA & 0.455 \\
& Temperature & One way ANOVA & 0.000 \\
& & Aw & One way ANOVA & 0.010 \\
& & Temperature $\times \mathrm{a}_{\mathrm{w}}$ & Two way ANOVA & 0.004 \\
\hline
\end{tabular}

(Samapundo et al., 2007). It is also generally well agreed that, in contrast to bacterial growth, $\mathrm{a}_{\mathrm{w}}$ is the most significant factor controlling fungal growth (Samapundo et al., 2007). Despite the fact that $\mathrm{a}_{\mathrm{w}}$ is usually considered the most influential factor on fungal growth, in the present study a significant influence of temperature on growth of both fungi was found, but no significant effect of $\mathrm{a}_{\mathrm{w}}$ or interaction temperature $\times a_{w}$ was detected. This could be explained by the low range of $a_{w}$ used ( 0.95 and 0.98 ) which are both good conditions for $P$. nordicum and $A$. westerdijkiae growth. However, the significant difference between 15 and $20^{\circ} \mathrm{C}$ must depend on the optimum condition for $A$. westerdijkiae and $P$. nordicum. In fact, A. westerdijkiae is more adapted to temperate climates, while $P$. nordicum is associated with colder climates, and this is apparent from our results, where $A$. westerdijkiae showed significantly higher growth at $20{ }^{\circ} \mathrm{C}(7 \mathrm{~cm})$ than at $15{ }^{\circ} \mathrm{C}(3.9 \mathrm{~cm})$, while $P$. nordicum showed no growth limitations for both temperatures.

A significant influence of temperature, $a_{w}$ and interaction temperature $\times \mathrm{a}_{\mathrm{w}}$ was found for OTA production by $A$. westerdijkiae, being $3 \% \mathrm{NaCl}$ at $20{ }^{\circ} \mathrm{C}$ the optimum condition. The significant influence of salt/ $\mathrm{a}_{\mathrm{w}}$ was expected, since OTA biosynthesis is naturally activated under sub-optimal, weak stress conditions (3\% salt) (Schmidt-Heydt, Baxter, Geisen, \& Magan, 2007).

\subsection{Influence of the matrix}

Based on the unexpected results described in section 3.1 further experiments were conducted to confirm the inability of both fungi to produce OTA in sausage-based medium, and the effect of matrix 
on OTA induction by test microorganisms. For that, different matrices were tested.

The results of $P$. nordicum and $A$. westerdijkiae growth in Ham, Ind and Trad with $3 \% \mathrm{NaCl} / 0.95 \mathrm{a}_{\mathrm{w}}$ incubated at $20{ }^{\circ} \mathrm{C}$ are represented in Figs. 3-5. It must be remarked that, for this particular study, $R$. mucilaginosa showed limited growth in Ham, either alone or in the Mix (C. zeylanoides $+R$. mucilaginosa), for both $P$. nordicum and $A$. westerdijkiae co-cultures. Based on this fact, results obtained for these conditions are presented but were not subject of analysis, as they would approximate to an axenic culture of the fungus.

Yeasts and starter significantly inhibited $P$. nordicum growth in all media except in in Trad with starter. On the other hand, A. westerdijkiae growth was significantly stimulated by the presence of starter, whereas significant decrease was exercised by yeasts in all media.

Results of OTA production by $P$. nordicum and $A$. westerdijkiae in the three media with $3 \% \mathrm{NaCl}$ incubated at $20^{\circ} \mathrm{C}$ are represented in Fig. 6. As reported for the previous test (section 3.1), P. nordicum was not able to produce detectable amounts of OTA in Ind, and the same happened in Trad, for all conditions tested. However, when in axenic culture, it produced the toxin in $\operatorname{Ham}(123 \mathrm{ng} / \mathrm{g}$ ), the same as previously reported by Vipotnik et al. (2017). In this matrix, C. zeylanoides significantly inhibited OTA production by P. nordicum, while the starter culture strongly stimulated its production. C. zeylanoides had been previously reported to have strong inhibitory effect on $P$. nordicum growth and OTA production on hambased media (Andrade et al., 2014; Simoncini et al., 2014) and on other synthetic salt-rich media (Nuñez et al., 2015; Virgili et al., 2012). These studies have all been conducted with single biocontrol agents and, to our knowledge, no studies have determined the effect of mixed agents, as is the case of the mix of yeasts and the starter culture used in this study (which is a mix of microorganisms composed of yeasts and bacteria). For $P$. nordicum, it seems like the starter, although reducing growth - or probably because of it, as previously mentioned -, stimulates fungal secondary metabolism and consequent OTA production.

A different behaviour was found for $R$. mucilaginosa, C. zeylanoides and starter co-inoculated with A. westerdijkiae. While yeasts significantly reduced fungal growth, OTA production was highly and significantly stimulated in all culture media. $R$. mucilaginosa, even though generally showing less effect on growth inhibition than $C$. zeylanoides, exerted a stronger effect on OTA stimulation. Starter culture also stimulated OTA production, with a stronger effect on both sausage-based media than on Ham.

The statistical analysis of the influence of three different matrices on fungal growth and OTA production are presented in Table 2. The analysis of the matrix influence revealed that $P$. nordicum was matrix-sensitive, with growth being significantly higher in Ham. The strain of P. nordicum used in this study has been isolated from ham, and maybe sausage composition includes one or more compounds which are inhibitory to $P$. nordicum ability to produce OTA. On the other hand, A. westerdijkiae, although also isolated from ham, showed similar growth in all media.

In terms of OTA production, the inability of $P$. nordicum to produce OTA in Ind found in the preliminary test was confirmed also for Trad. For A. westerdijkiae the matrix effect, although not significant, can also be observed for yeast and starter treatments. The production of OTA was always higher in Trad than in Ind, maybe due to the fact that industrial sausage has more anti-fungal preservatives added than traditional sausage. While yeasts generally enhanced OTA more strongly in Ham, starter showed stronger effect on both sausage-based media. This difference may be a result of starter being more adapted to sausage than to ham matrix, since it is a culture commercially used in sausage fermentation.

It would be interesting to determine and compare the chemical composition of these three meat products throughout the ripening

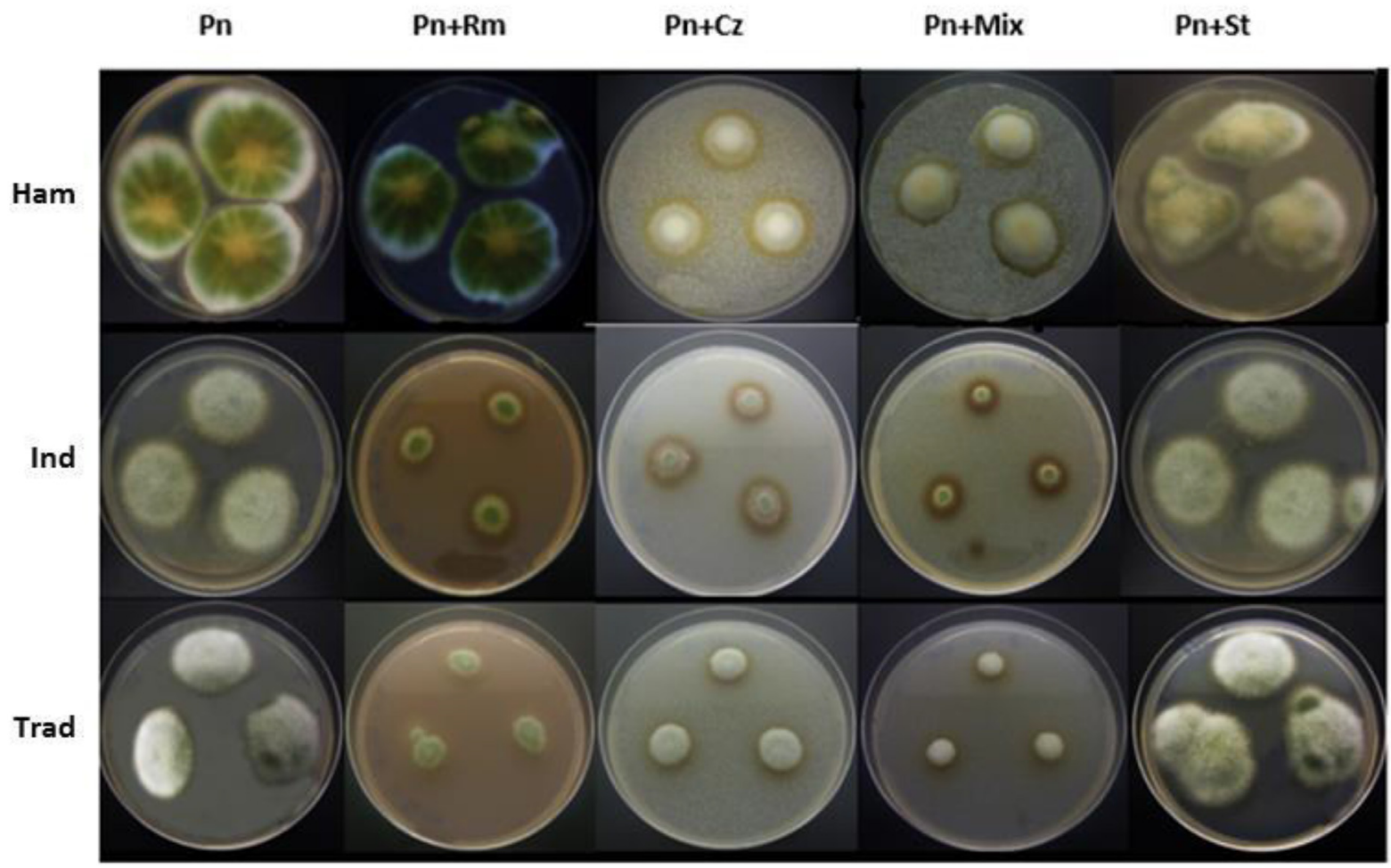

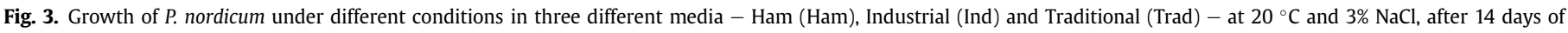
incubation. Pn: P. nordicum; Rm: R. mucilaginosa; Cz: C. zeylanoides; Mix: mix of the two yeasts; St: starter culture. 


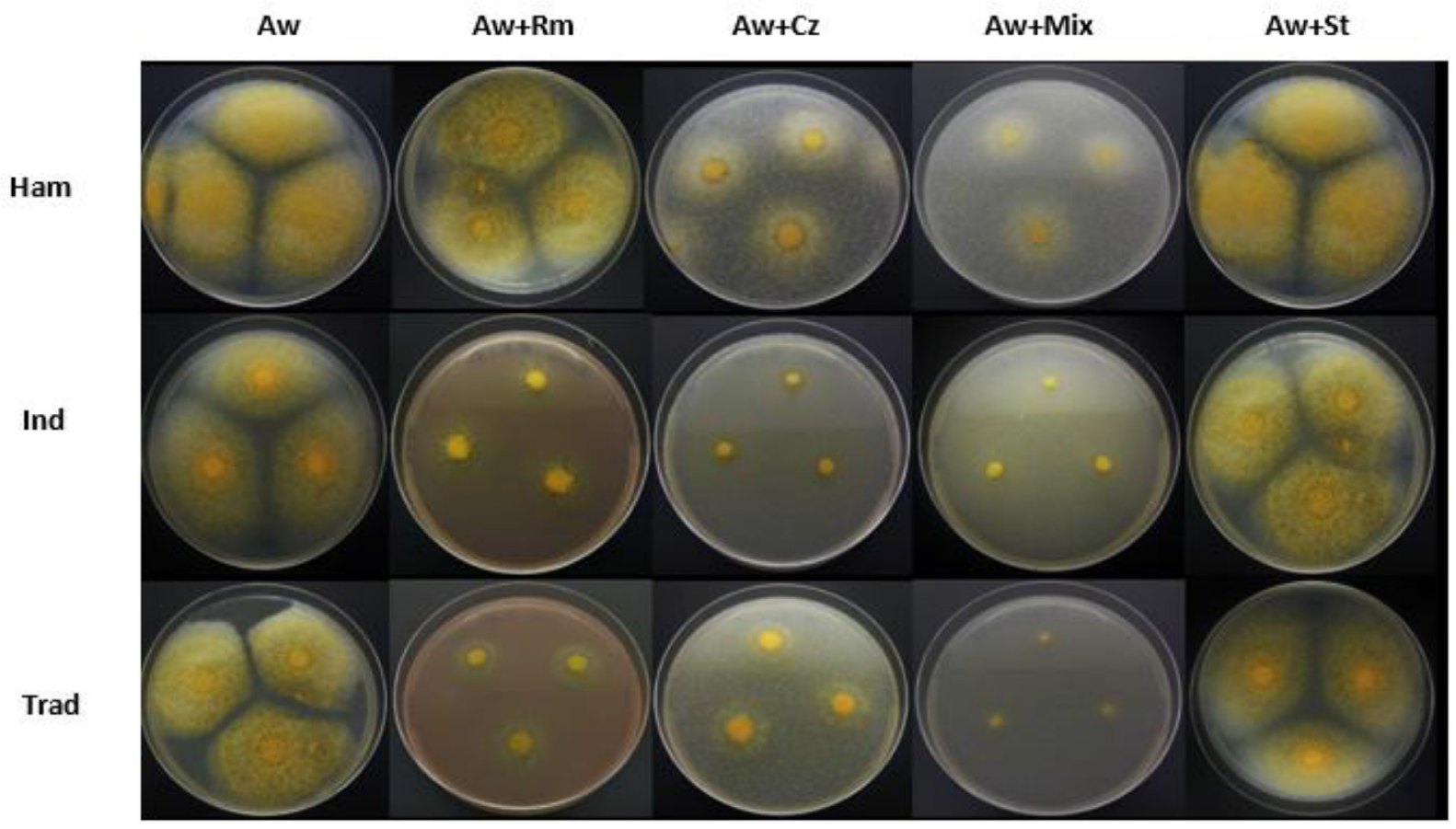

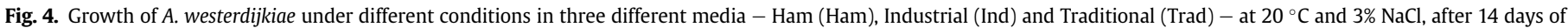
incubation. Aw: A. westerdijkiae; Rm: R. mucilaginosa; Cz: C. zeylanoides; Mix: mix of the two yeasts; St: starter culture.

\section{P. nordicum}

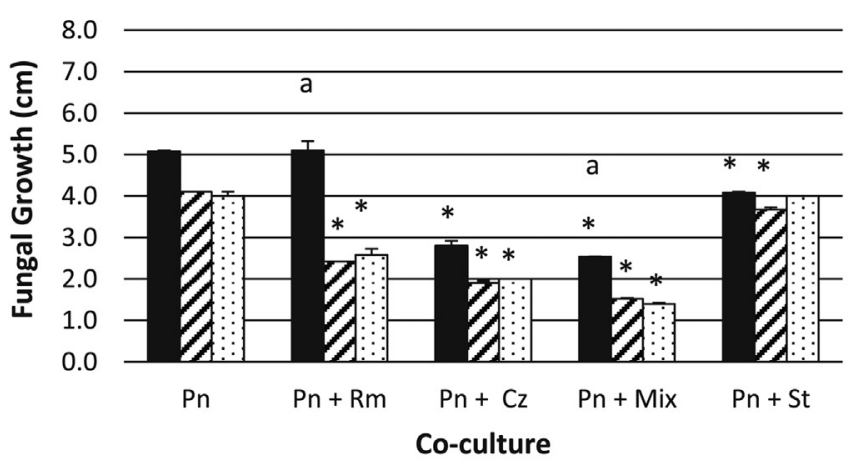

A. westerdijkiae

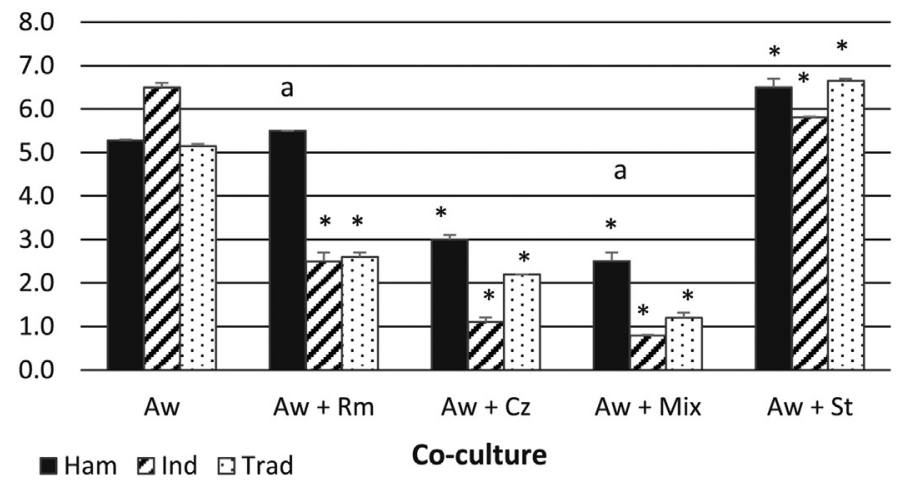

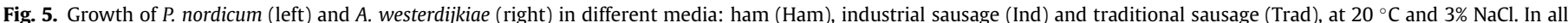

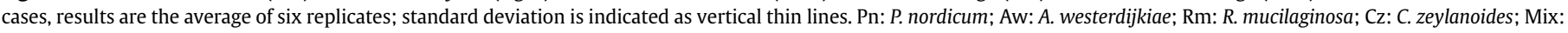
mix of the two yeasts; St: starter culture.

*Significantly different from control (fungus only), $p \leq 0.05$.

a no growth of $R$. mucilaginosa.

${ }^{\mathrm{b}}$ no growth of $R$. mucilaginosa; effect probably limited to C. zeylanoides.

period, which is out of the scope of this study. The acknowledgment of the mechanism behind this effect could support the development of a OTA prevention method. Further studies are needed in this field of research.

\section{Conclusions}

Even though $P$. nordicum is considered fully adapted to protein and salt-rich matrices like processed meat products, it seems to be strongly influenced by the matrix. The fungus was not able to produce OTA in sausage-based media, probably due to the chemical composition of this matrix (preservatives, condiments, etc). In this case, it would be of major interest to study chemical compounds from these matrices that could be responsible for this effect, and thus be used as OTA control agents.

On the other hand, A. westerdijkiae, although not generally associated with meat products, was not significantly influenced by the matrix, and showed to be capable of producing high amounts of OTA in all media tested. It has been demonstrated that this fungus should also be subject of interest in future works dealing with OTA contamination of meat products.

Yeasts were able to inhibit $P$. nordicum ability to produce OTA in ham, but unexpectedly OTA production was strongly stimulated by the starter culture. In $A$. westerdijkiae, yeasts and starter 


\section{P. nordicum}

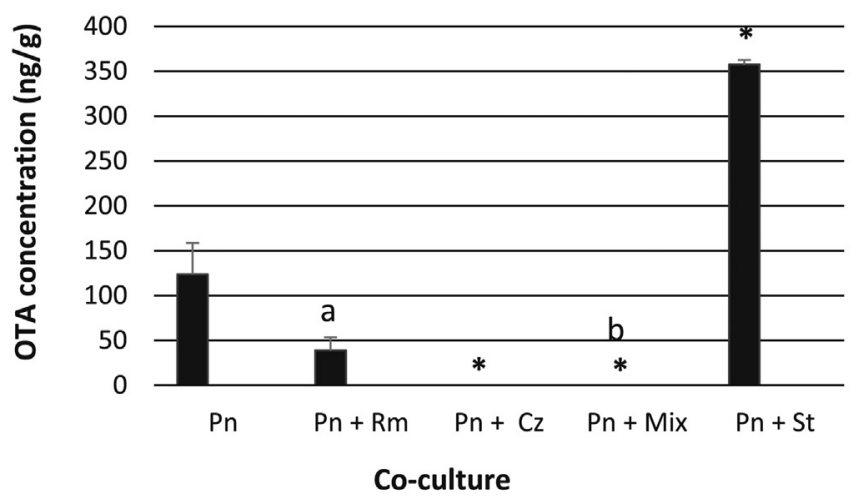

A. westerdijkiae

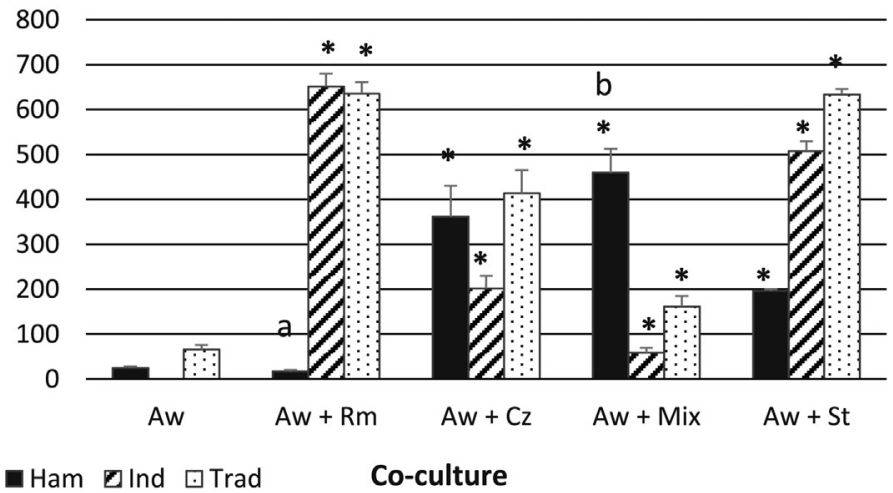

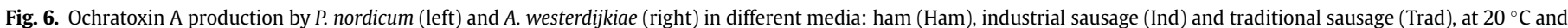
$3 \% \mathrm{NaCl}$. In all cases, results are the average of six replicates; standard deviation is indicated as vertical thin lines.

Pn: P. nordicum; Aw: A. westerdijkiae; Rm: R. mucilaginosa; Cz: C. zeylanoides; Mix: mix of the two yeasts; St: starter culture.

*Significantly different from control (fungus only), $p \leq 0.05$.

a no growth of $R$. mucilaginosa.

${ }^{\mathrm{b}}$ no growth of $R$. mucilaginosa; effect probably limited to C. zeylanoides.

Table 2

Statistical analyses of the influence of matrix on P. nordicum (Pn) and A. westerdijkiae (Aw) growth and OTA production $(p<0.05)$.

\begin{tabular}{lllll}
\hline Species & Test variable & Factor & Statistical set & $p$-value \\
\hline Pn & Growth & Ham-Ind & One way ANOVA & 0.000 \\
& & Ham-Trad & One way ANOVA & 0.000 \\
& \multirow{3}{*}{ OTA } & Ind-Trad & One way ANOVA & 0.982 \\
& & Ham-Ind & One way ANOVA & 0.002 \\
& Ham-Trad & One way ANOVA & 0.002 \\
& \multirow{3}{*}{ Growth } & Ind-Trad & One way ANOVA & 1.000 \\
& & Ham-Ind & One way ANOVA & 0.053 \\
& \multirow{3}{*}{ OTA } & Ham-Trad & One way ANOVA & 0.154 \\
& & Ind-Trad & One way ANOVA & 0.877 \\
& & Ham- Ind & One way ANOVA & 0.678 \\
& & Ham-Trad & One way ANOVA & 0.127 \\
& & Ind-Trad & One way ANOVA & 0.490 \\
\hline
\end{tabular}

significantly enhanced OTA production. Yeasts and starter culture used in this study revealed contrasting effects on the two ochatoxigenic fungi, showing that the study of biocontrol agents against mycotoxigenic fungi must involve the microbial community instead of isolated microorganisms.

\section{Acknowledgements}

This work was supported by the Foundation for Science and Technology (FCT, Portugal) and FEDER under Programme PT2020 for financial support to CIMO [UID/AGR/00690/2013].

\section{References}

Abrunhosa, L., Inês, A., Rodrigues, A. I., Guimarães, A., Pereira, V. L., Parpot, P., et al. (2014). Biodegradation of ochratoxin A by Pediococcus parvulus isolated from Douro wines. International Journal of Food Microbiology, 188, 45-52.

Andrade, M. J., Rodriguez, M., Casado, E. M., Bermudez, E., \& Cordoba, J. J. (2010). Effect of selected strains of Debaryomyces hansenii on the volatile compound production of dry fermented sausage "salchichón”. Meat Science, 85, 256-264.

Andrade, M. J., Thorsen, L., Rodríguez, A., Córdoba, J. J., \& Jespersen, L. (2014). Inhibition of ochratoxigenic moulds by Debaryomyces hansenii strains for biopreservation of dry-cured meat products. International Journal of Food Microbiology, 170, 70-77.

Boudra, H., Le Bars, P., \& Le Bars, J. (1995). Thermostability of ochratoxin A in wheat under two moisture conditions. Applied Environmental Microbiology, 61, 1156-1158.

Bragulat, M. R., Abarca, M. L., \& Cabañes, F. J. (2001). An easy screening method for fungi producing ochratoxin A in pure culture. International Journal of Food
Microbiology, 71, 139-144.

Cadavez, V., Gonzales-Barron, U., Pires, P., Fernandes, E., Pereira, A. P., Gomes, A., et al. (2016). An assessment of the processing and physicochemical factors contributing to the microbial contamination of salpicão, a naturally-fermented Portuguese sausage. LWT-Food Science and Technology, 72, 107-116.

Day, A., Schneider, C., \& Schneider, B. L. (2004). Chapter 6-yeast cell synchronization. Methods in Molecular Biology, 241, 55-76.

EFSA. (2009). Scientific report submitted to EFSA (European Food Safety Authority). Review of mycotoxin-detoxifying agents used as feed additives: Mode of action, efficacy and feed/food safety. Available at: http://www.efsa.europa.eu/en/ supporting/pub/22e.htm.

Escher, F. E., Koehler, P. E., \& Ayres, J. C. (1973). Production of ochratoxins A and B on country cured ham. Applied Microbiology, 26, 27-30.

Fialho, M. B., Toffano, L., Pedroso, M. P., Augusto, F. \& Pascholati, S. F. (2009). Volatile organic compounds produced by Saccharomyces cerevisiae inhibit the in vitro development of Guignardia citricarpa, the causal agent of citrus black spot. World Journal of Microbiology and Biotechnology, 2, 925-932.

Flores, M., Corral, S., Cano-García, L., Salvador, A., \& Belloch, C. (2015). Yeast strains as potential aroma enhancers in dry fermented sausages. International Journal of Food Microbiology, 212, 16-24.

Gerez, C. L., Dallagnol, A., Ponsone, L., Chulze, S., \& Valdez, G. F. (2014). Ochratoxin A production by Aspergillus Niger: Effect of water activity and a biopreserver formulated with Lactobacillus plantarum CRL 778. Food Control, 45, 115-119.

Gil-Serna, J., Patiño, B., Cortés, L., González-Jaén, M. T., \& Vazquez, C. (2011). Mechanisms involved in reduction of ochratoxin A produced by Aspergillus westerdijkiae using Debaryomyces hansenii CYC 1244. International Journal of Food Microbiology, 151, 113-118.

Gil-Serna, J., Patiño, B., Cortés, L., González-Jaén, M. T., \& Vazquez, C. (2015). Aspergillus steynii and Aspergillus westerdijkiae as potential risk of OTA contamination in food products in warm climates. Food Microbiology, 46, $168-175$.

Gonzales-Barron, U. Cadavez, V., Pereira, A. P., Gomes, A. Araújo, J. P., Saavedra, M. J., et al. (2015). Relating microbiological and physicochemical patterns of a traditional Portuguese fermented sausage along processing. Food Research International, 78, 50-61.

Hernández-Montiel, L. G., Ochoa, J. L., Troyo-Diéguez, E., \& Larralde-Corona, C. P. (2010). Biocontrol of postharvest blue mold (Penicillium italicum Wehmer) on Mexican lime by marine and citrus Debaryomyces hansenii isolates. Postharvest Biology and Technology, 56, 181-187.

Iacumin, L., Manzano, M., Andyanto, D., \& Comi, G. (2017). Biocontrol of ochratoxigenic moulds (Aspergillus ochraceus and Penicillium nordicum) by Debaryomyces hansenii and Saccharomycopsis fibuligera during speck production. Food Microbiology, 62, 88-195.

Iacumin, L., Milesi, S., Pirani, S., Comi, G., \& Chiesa, L. M. (2011). Ochratoxigenic mold and ochratoxin a in fermented sausages from different areas in northern Italy: Occurrence, reduction or prevention with ozonated air. Journal of Food Safety, 31, $538-545$.

Kurtzman, C. P., \& Robnett, C. J. (1998). Identification and phylogeny of ascomycetous yeasts from analysis of nuclear large subunit (26S) ribosomal DNA partial sequences. Antonie Van Leeuwenhoek, 73, 331-371.

Masih, E. I., \& Paul, B. (2002). Secretion of beta-1,3-glucanases by the yeast Pichia membranifaciens and its possible role in the biocontrol of Botrytis cinerea causing grey mold disease of the grapevine. Current Microbiology, 44, 391-395.

Masoud, W., Poll, L., \& Jakobsen, M. (2005). Influence of volatile compounds produced by yeasts predominant during processing of Coffea arabica in East Africa 
on growth and ochratoxin A (OTA) production by Aspergillus ochraceus. Yeast, 22, 1133-1142.

Mendonça, R. C. S., Gouvêa, D. M., Hungaro, H. M., Sodré, A. F., \& Querol-Simon, A. (2013). Dynamics of the yeast flora in artisanal country style and industrial dry cured sausage (yeast in fermented sausage). Food Control, 29, 143-148.

Ngang, J. J. E., Germaine, Y., Sylvain, L. S. Y., Kouebou, C. P., Fanche, S. A. Tsochi, D. L. K., et al. (2015). Antifungal properties of selected lactic acid bacteria and application in the biological control of ochratoxin A producing fungi during cocoa fermentation. Biocontrol Science and Technology, 25, 245-259.

Nielsen, D. S., Jacobsen, T., Jespersen, L., Koch, A. G., \& Arneborg, N. (2008). Occurrence and growth of yeasts in processed meat products - implications for potential spoilage. Meat Science, 80, 919-926.

Nuñez, F., Lara, M. S., Peromingo, L., Delgado, J., Sanchez-Montero, L., \& Andrade, M. J. (2015). Selection and evaluation of Debaryomyces hansenii isolates as potential bioprotective agents against toxigenic penicillia in dryfermented sausages. Food Microbiology, 46, 114-120.

Pereira, M., Beux, M., Pagnoncelli, B., Soccol, V. T., Rodrigues, C., \& Soccol, C. R (2016). Isolation, selection and evaluation of antagonistic yeasts and lactic acid bacteria against ochratoxigenic fungus Aspergillus westerdijkiae on coffee beans. Letters in Applied Microbiology, 62, 96-101.

Rodrigues, P., Santos, C., Venancio, A., \& Lima, N. (2011). Species identification of Aspergillus section Flavi isolates from Portuguese almonds using phenotypic, including MALDI-TOF ICMS, and molecular approaches. Journal of Applied Microbiology, 111, 877-892.

Rodrigues, P., Venancio, A., Kozakiewicz, Z., \& Lima, N. (2009). A polyphasic approach to the identification of aflatoxigenic and non-aflatoxigenic strains of Aspergillus Section Flavi isolated from Portuguese almonds. International Journal of Food Microbiology, 129, 187-193.

Rodriguez, A., Bernaldez, V., Rodriguez, M., Andrade, M. J., Nuñez, F., \& Cordoba, J. J. (2015). Effect of selected protective cultures on ochratoxin A accumulation in dry-cured Iberian ham during its ripening process. LWT-Food Science and Technology, 60, 923-928.

Romano, P., Capece, A., \& Jespersen, L. (2006). Taxonomic and ecological diversity of food and beverage yeasts. In A. Querol, \& G. H. Fleet (Eds.), The yeasts handbook (pp. 13-53). Germany: Springer Verlag.

Samapundo, S., Devlieghere, F., Geeraerd, A. H., De Meulenaer, B., Van Impe, J. F., \& Debevere, J. (2007). Modelling of the individual and combined effects of water activity and temperature on the radial growth of Aspergillus flavus and A. parasiticus on corn. Food Microbiology, 24, 517-529.

Samelis, J., \& Sofos, N. (2003). Yeasts in meat and meat products. In T. Boekhout, \& V. Robert (Eds.), Yeasts in food, beneficial and detrimental aspects (pp. 234-265). Germany: Behr's Verlag.

Scaramuzza, N., Diaferia, C., \& Berni, E. (2015). Monitoring the mycobiota of three plants manufacturing Culatello (a typical Italian meat product). International Journal of Food Microbiology, 203, 78-85.

Schmidt-Heydt, M., Baxter, E., Geisen, R., \& Magan, N. (2007). Physiological relationship between food preservatives, environmental factors, ochratoxin and otapksPV gene expression by Penicillium verrucosum. International Journal of Food Microbiology, 119, 277-283.

Schmidt-Heydt, M., Stoll, D., \& Geisen, R. (2013). Fungicides effectively used for growth inhibition of several fungi could induce mycotoxin biosynthesis in toxigenic species. International Journal of Food Microbiology, 166, 407-412.

Simoncini, N., Virgili, R., Spadola, G., \& Battilani, P. (2014). Autochthonous yeasts as potential biocontrol agents in dry-cured meat products. Food Control, 46, $160-167$.

Suarez, V. B., Frison, L., de Basilico, M. Z., Rivera, M., \& Reinheimer, J. (2005) Inhibitory activity of phosphates on moulds isolated from foods and food processing plants. Journal of Food Protection, 68, 2475-2479.

Suarez, V. B., Tremmel, G. J., Rivera, M., Reinheimer, J. A., \& Meinardi, C. A. (2012). Polyphosphates as inhibitors of surface mould growth on hard cheese during ripening. International Journal of Dairy Technology, 65, 410-415.

Taczman-Brückner, A., Mohácsi-Farkas, C., Balla, C., \& Kiskó, G. (2005). Mode of action of Kluyveromyces lactis in biocontrol of Penicillium expansum. Acta Alimentaria Hungarica, 34, 153-160.

Taverniers, I., De Loose, M., \& Van Bockstaele, E. (2004). Trends in quality in the analytical laboratory. II. Analytical method validation and quality assurance. Trends in Analytical Chemistry, 23, 535-552.

Vipotnik, Z., Rodríguez, A., \& Rodrigues, P. (2017). Aspergillus westerdijkiae as a major ochratoxin A risk in dry-cured ham based-media. International Journal of Food Microbiology, 241, 244-251.

Virgili, R., Simoncini, N., Toscani, T., Leggieri, M. C., Formenti, S., \& Battilani, P. (2012). Biocontrol of Penicillium nordicum growth and ochratoxin a production by native yeasts of dry cured ham. Toxins, 4, 68-82. 